\title{
FOURIER INVERSION FORMULA FOR DISCRETE NILPOTENT GROUPS
}

\author{
TSUYOSHI KAJIWARA
}

(Received 23 June 1987)

Communicated by W. Moran

\begin{abstract}
Let $G$ be a countable torsion free finitely generated nilpotent group. Then the Fourier transform can be considered as a map from the space of bounded degree 1 random operators to the Fourier algebra $A(G)$. In this paper, we recover the matrix elements of a positive random variable from the corresponding positive definite function in $A(G)$ for such a group.
\end{abstract}

1980 Mathematics subject classification (Amer. Math. Soc.): 46 L 55.

\section{Introduction}

When $G$ is a locally compact abelian group, the Fourier transform is a map from $L^{1}(G)$ and the inversion formula can be expressed in the same form. More generally, let $G$ be a type I group. As in Paterson [2], the Fourier transform is considered as a map from the $L^{1}$-space on $\hat{G}$ with respect to a Plancherel measure to the Fourier algebra $A(G)$. In [2], Paterson writes down the explicit form of the Fourier inversion formula in this sense when $G$ is the Heisenberg Lie group.

On the other hand, for general groups not of type I, there is no good Plancherel formula based on $\hat{G}$ in a classical sense. Recently in [4], Sutherland considered the Plancherel formula of some groups not of type I based on $\hat{G}$ using the "singular integration theory" of Connes [1].

In this paper, we extend the Connes-Sutherland theory to the non-split groups. In this case, our Fourier transform is a map from the space of

(C) 1989 Australian Mathematical Society 0263-6115/89 \$A2.00+0.00 
"bounded" degree 1 random operators to $A(G)$. The main purpose of this paper is to recover the matrix elements of a positive random variable from the corresponding positive definite function in $A(G)$ for a finitely generated torsion free nilpotent group $G$. This can be considered as the Fourier inversion formula of such groups.

I would like to express my hearty thanks to Dr. S. Yamagami for fruitful discussions and to Professor $O$. Takenouchi for his constant encouragement and good advice.

\section{General theory}

Let $G$ be a separable unimodular locally compact group with a normal maximal abelian subgroup $N$. We assume that we can choose a suitable $G$ invariant conull subset $\Lambda$ of $\hat{N}$ such that $\operatorname{Ind}_{N \uparrow G} \chi$ is irreducible for every $\chi \in \Lambda$. Since the above group extension does not split in general, it is not appropriate to consider the groupoid $\hat{N} \times G / N$. But even in this case, we can consider "singular integration theory" analogous to that in Connes [1].

Take $\alpha \in \Lambda$. We use "right" notation. We write $L^{2}(G, N, \alpha)$ for the representation space of $\operatorname{Ind}_{N \uparrow G} \alpha$. An element $\xi$ in $L^{2}(G, N, \alpha)$ satisfies $\xi(n \cdot g)=$ $\langle n, \alpha\rangle \xi(g)$ for $n \in N, g \in G$, and $\operatorname{Ind}_{N \uparrow G}(g)$ acts on $L^{2}(G, N, \alpha)$ as a right translation operator. We denote by $\lambda$ the right regular representation of $G$ on $L^{2}(G)$. Let $U^{\alpha}=\operatorname{Ind}_{N \uparrow G} \alpha$. Take a Haar measure $\mu$ on $\hat{N}$ and abbreviate $d \mu(\alpha)$ to $d \alpha$. Then we have the following irreducible decomposition:

$$
\lambda(g)=\int_{\Lambda}^{\oplus} U^{\alpha}(g) d \alpha .
$$

This is just the ordinary partial Fourier-Plancherel transform with respect to $N$.

We assume the action of $G / N$ on $\hat{N}$ leaves $\mu$ invariant. Then we can consider some unimodular groupoid like structure. By using this, we extend Connes' theory to our situation. Let $\left\{T^{\alpha}\right\}_{\alpha \in \Lambda}$ be a measurable family of closed operators on $L^{2}(G, N, \alpha)$. We define an isometric operator $V^{\alpha}(g)$ from $L^{2}(G, N, \alpha)$ to $L^{2}(G, N, g \cdot \alpha)$ for $\xi \in L^{2}(G, N, \alpha), g \in G$, by $\left(V^{\alpha}(g) \xi\right)\left(g^{\prime}\right)=$ $\xi\left(g^{-1} g^{\prime}\right)$ for every $g^{\prime} \in G$.

Definition 1. A measurable field $\left\{T^{\alpha}\right\}_{\alpha \in \Lambda}$ is a random operator if

$$
V^{\alpha}(g) T^{\alpha}=T^{g \cdot \alpha} V^{\alpha}(g)
$$

for all $g \in G$. 
Two random operators $\left\{T^{\alpha}\right\}_{\alpha \in \Lambda}$ and $\left\{T^{\alpha}\right\}_{\alpha \in \Lambda}$ are called equivalent if $T^{\alpha}=T^{\prime \alpha}$ holds on some $G$-invariant conull subset of $\Lambda$. A random operator $\left\{T^{\alpha}\right\}_{\alpha \in \Lambda}$ is called bounded (positive) if essup $\left\|T^{\alpha}\right\|<\infty\left(T^{\alpha} \geq 0\right)$ on some $G$-invariant conull subset of $\Lambda$. We write $\operatorname{End}_{\mu}(G)$ for the set of all equivalence classes of bounded random operators. When $G$ is in the form of a semi-direct product of $N$ by $G / N$, this definition coincides with that of Connes [1], and the Haar measure $\mu$ on $\hat{N}$ corresponds to the transverse measure.

Analogously to Connes [1], for every positive random operator $T=$ $\left\{T^{\alpha}\right\}_{\alpha \in \Lambda}$ we can construct a normal weight $\varphi_{T}$ on $\operatorname{End}_{\mu}(G)$. We denote by $\mathrm{M}^{+}(G / N)$ the set of non-negative valued measurable functions on $G / N$. For $f$ in $M^{+}(G / N)$ we write $M_{f}$ for the pointwise multiplication operator on $L^{2}(G, N \alpha)$ given by $f$. For a positive self adjoint operator $H$ on some Hilbert space $\nVdash$, we use $\omega_{H}$ to denote the normal weight given by $H$ on $B(\not)$ (cf. Pedersen-Takesaki [3]).

We fix $A \in \operatorname{End}_{\mu}(G)^{+}$. For $f \in M^{+}(G / N)$ consider the map

$$
f \rightarrow \omega_{T}\left(\left(A^{\alpha}\right)^{1 / 2} M_{f}\left(A^{\alpha}\right)^{1 / 2}\right) .
$$

This map gives positive measures on $G / N$. By the randomness of $\left\{T^{\alpha}\right\}_{\alpha \in \Lambda}$ and $\left\{A^{\alpha}\right\}_{\alpha \in \Lambda}$, this field satisfies some covariance condition similar to that in Connes [1]. Take $f$ in $M^{+}(G / N)$ such that $\int_{G / N}^{\oplus} f(\dot{g}) d \dot{g}=1$. We call this $f$ a partition of unity. Then the value

$$
\int_{\hat{N}}^{\oplus} \omega_{H}\left(\left(A^{\alpha}\right)^{1 / 2} M_{f}\left(A^{\alpha}\right)^{1 / 2}\right) d \alpha
$$

is independent of the choice of $f$. We write $\varphi_{H}(A)$ for this integral. Then the map $A \rightarrow \varphi_{T}(A)$ (for each $A \in \operatorname{End}_{\mu}(G)^{+}$) gives a normal weight on $\operatorname{End}_{\mu}(G)$. Similarly to Connes [1], we have the following result.

LEMMA 2. For every weight $\varphi$ on $\operatorname{End}_{\mu}(G)$, there exists a positive random operator $\left\{T^{\alpha}\right\}_{\alpha \in \Lambda}$ such that $\varphi=\varphi_{T}$.

Proof. We define a unitary operator $V(g)$ on $\int_{\tilde{N}}^{\oplus} L^{2}(G, N, \alpha) d \alpha$, for $\xi \in$ $\int_{\hat{N}}^{\oplus} L^{2}(G, N, \alpha) d \alpha$, by $(V(g) \xi)\left(g^{\prime}, \alpha\right)=\xi\left(g^{-1} g^{\prime}, \alpha\right)\left(g^{\prime} \in G, \alpha \in \hat{N}\right)$. Then $V(n)$ is equal to $\int_{\hat{N}}^{\oplus} M_{\langle n, \alpha\rangle} d \alpha$ for $n \in N$. For every $A$ in $\operatorname{End}_{\mu}(G)$, the map $g \rightarrow \operatorname{Ad} V(g)(A)$ is right $N$ invariant. Take $B$ in $\int_{\hat{N}}^{\oplus} B\left(L^{2}(G, N, \alpha)\right)^{+} d \alpha$. We define $W(B)$ in $\operatorname{End}_{\mu}(G)^{+}$by $\int_{G / N}^{\oplus} \operatorname{Ad} V(g)(B) d \dot{g}$. Then this $W$ is an operator valued weight from $\int_{\hat{N}}^{\oplus} B\left(L^{2}(G, N, \alpha)\right) d \alpha$ to $\operatorname{End}_{\mu}(G)$. 
Since $\varphi \circ W$ is a weight on $\int_{\hat{N}}^{\oplus} B\left(L^{2}(G, N, \alpha)\right) d \alpha$, there exists a measurable family of positive self adjoint operators $\left\{T^{\alpha}\right\}_{\alpha \in \Lambda}$ such that $\varphi \circ W=$ $\int_{\hat{N}}^{\oplus} \omega_{T^{\alpha}} d \alpha$. For $A$ in $\operatorname{End}_{\mu}(G)^{+}$and $f$ in $M^{+}(G / N)$ with $\int_{G / N} f(\dot{g}) d \dot{g}=1$, we have

$$
W\left(\int_{\hat{N}}^{\oplus}\left(A^{\alpha}\right)^{1 / 2} M_{f}\left(A^{\alpha}\right)^{1 / 2} d, \alpha\right)=A
$$

By using this, we have this lemma.

The formula in the above lemma can be considered as the "singular" integral decomposition on $\hat{N}$ of a weight $\varphi$. We write this fact symbolically as $\varphi=\int_{\hat{N} / \sim} \operatorname{Tr}\left(T^{\alpha}\right), d \Lambda$. We apply this lemma to the Haar weight $\psi$ on $\operatorname{End}_{\mu}(G)$, and have the following "singular Plancherel fomrula" of $G$.

Proposition 3. For normalized Haar measures on $\hat{N}$ and $G / N, \psi(A)=$ $\int_{\hat{N} / \sim}^{\oplus} \operatorname{Tr}(1 \cdot) d \alpha$.

This formula is a group extension version of that in Sutherland [4], and shows the possibility of normalizing Plancherel measures of some groups not of type $I$.

We denote the Fourier algebra of $G$ by $A(G)$. Then $A(G)$ is canonically isomorphic to the predual of $\lambda(G)^{\prime \prime} \simeq \operatorname{End}_{\mu}(G)$, and a positive random operator $\left\{T^{\alpha}\right\}_{\alpha \in \Lambda}$ such that $\varphi_{T}(1)<\infty$ gives rise to a positive definite function in $A(G)$. When $G$ is equal to $N$ and abelian, this map is the ordinary Fourier transformation from $L^{1}(G)$ to $A(G)$. We investigate the inverse of the above transform in the special case in the next section. We remark that Paterson [2] solved this type of problem in the case of the Heisenberg Lie group.

\section{Discrete nilpotent groups}

Let $G$ be a countable torsion free finitely generated nilpotent group. Take an abelian normal subgroup $N$ which is maximal in the set of normal abelian subgroups of $G$.

LEMMA 4. $N$ is a maximal abelian subgroup of $G$.

Proof. Let $M$ be the centralizer of $N$ in $G$. Suppose that $M \neq N . G$ acts on $M / N$ via inner automorphism. By the nilpotency of $G$, there exists a fixed point $\dot{h} \neq \dot{e}$. Take a representative $h$ of $\dot{h}$ in $G$. Then, the subgroup generated by $N$ and $h$ is normal, abelian and strictly larger than $N$. 
Fix some normal maximal abelian subgroup in $G$, and denote this by $N$. Take $\chi \in \hat{N}$. Then $\operatorname{Ind}_{N \uparrow G} \chi$ is irreducible if and only if $g \cdot \chi=\chi$ implies $g \in N$. Let $\mu$ be a Haar measure in $\hat{N}$.

LEMMA 5. There exists a G-invariant $\mu$-conull subset $\Lambda$ in $\hat{N}$ such that for every $\chi$ in $\hat{N}, g \cdot \chi=\chi$ implies $g \in N$.

Proof. $N$ is of the form $\mathbf{Z}^{r}$, and $\hat{N}$ is isomorphic to $\mathbf{T}^{r}$. We put coordinates on $\hat{N}$ and represent $\chi \in N$ as $\chi=\left(\chi_{1}, \ldots, \chi_{r}\right)$. Then $g \cdot \chi=\chi$ implies $\left\langle\chi, g^{-1} n-n\right\rangle=0$ for every $n$ in $N$. We denote the $i$ th component of $n$ by $(n)_{i}$. By using this notation, we have $\sum_{i=1}^{r} \chi_{i}\left(g^{-1} n-n\right)_{i}=0$ for every $n$ in $N$. There exists a conull subset $\Lambda^{\prime}$ in $\hat{N}$ such that for all $\left(\chi_{1}, \ldots, \chi_{r}\right) \in$ $\Lambda^{\prime}, 1, \chi_{1}, \ldots, \chi_{r}$ are rationally independent. Let $N^{\prime}=\left\{g \in G ;\left(g^{-1} n-n\right)_{i}=0\right.$ for ever $n \in N$ \}. Then $N^{\prime} \supset N$. Take $g \in N^{\prime}$. Then $g \cdot \chi=\chi$ holds for almost all $\chi$. By the continuity of the action of $G$ on $\hat{N}, g \cdot \chi=\chi$ for all $\chi \in \hat{N}$. This shows that $g$ is contained in $N$.

\section{Inversion formula}

By the result in Section 2, we can use the method in Section 1 when $G$ is a countable torsion free finitely generated nilpotent group. Take a cross section set $X \subset G$ for the quotient map from $G$ to $G / N$ such that $e \in X$. Every $g \in G$ can be written uniquely as $g=n \cdot x(n \in N, x \in X)$. We define an element $\xi_{x}^{\alpha}$ in $L^{2}(G, N, \alpha)$ by $\xi_{x}^{\alpha}(g)=\langle\alpha, n\rangle$ when $\dot{g}=\dot{x}$ and $g=n \cdot x$ ( $n \in N, x \in X$ ), and $\xi_{x}^{\alpha}(g)=0$ otherwise. Then, $\left\{\xi_{x}^{\alpha}\right\}_{\dot{x} \in G / N}$ is a CONS of $L^{2}(G, N, \alpha)$.

By using this CONS, we define matrix elements of random operators. When a random operator $\left\{B^{\alpha}\right\}_{\alpha \in \Lambda}$ is bounded, put $B_{x, y}^{\alpha}=\left\langle B^{\alpha} \xi_{y}^{\alpha}, \xi_{x}^{\alpha}\right\rangle$. When a random operator $\left\{B^{\alpha}\right\}_{\alpha \in \Lambda}$ is positive self adjoint, put

$$
\left.B_{x, y}^{\alpha}=\left\langle\left(B^{\alpha}\right)^{1 / 2} \xi^{\alpha}\right) y,\left(B^{\alpha}\right)^{1 / 2} \xi_{x}^{\alpha}\right\rangle
$$

if $\xi_{x}^{\alpha}$ and $\xi_{y}^{\alpha}$ are contained in $D\left(\left(B^{\alpha}\right)^{1 / 2}\right)$. When $\left\{B^{\alpha}\right\}_{\alpha \in \Lambda}$ is bounded and positive, the two definitions coincide. For a positive self adjoint operator $H$ on a Hilbert space $\mathcal{H}$ and a positive number $\varepsilon$, denote $H(1+\varepsilon H)^{-1}$ by $H_{\varepsilon}$.

LEMMA 6. Let $\left\{T^{\alpha}\right\}_{\alpha \in \Lambda}$ be a positive random operator. If $\varphi_{T}(1)<\infty$, we can take a $G$ invariant conull subset $\Lambda^{\prime}$ of $\Lambda$ such that for every $\alpha$ in $\Lambda^{\prime}$ all matrix elements of $T^{\alpha}$ with respect to $\left\{\xi_{x}^{\alpha}\right\}_{\dot{x} \in G / N}$ exist. 
Proof. Since the value of $\varphi_{T}$ is independent of the choice of $f$, we may choose $f$ to be the characteristic function of $\dot{x}$. Then, $M_{f}$ is the projection $p_{\dot{x}}$ on the 1-dimensional subspace generated by $\xi_{x}^{\alpha}$.

Then $\varphi_{T}(1)=\int_{\hat{N}} \omega_{T^{n}}\left(p_{\dot{e}}\right) d \alpha$. From this there exists $\Lambda^{\prime}$ such that $\omega_{T^{a}}\left(p_{\dot{e}}\right)$ $<\infty$ for $\alpha \in \Lambda^{\prime}$. By the definition of $\omega_{T^{\alpha}}$,

$$
\lim _{\varepsilon \rightarrow 0}\left\|\left(T^{\alpha}\right)_{\varepsilon}^{1 / 2} \xi_{x}^{\alpha}\right\|<\infty .
$$

This shows that $\xi_{x}^{\alpha}$ is contained in the domain of $\left(T^{\alpha}\right)^{1 / 2}$ for every $\dot{x}$ in $G / N$.

As in the proof of the above lemma, for every $H$ in $B\left(L^{2}(G, N, \alpha)\right)^{+}$, $\omega_{T^{\alpha}}\left(H^{1 / 2} p_{\dot{x}} H^{1 / 2}\right)<\infty$ is equivalent to $H^{1 / 2} \xi_{x}^{\alpha} \in D\left(\left(T^{\alpha}\right)^{1 / 2}\right)$. When $\varphi_{T}(1)<$ $\infty, \varphi_{T}(A)$ has meaning for every $A \in \operatorname{End}_{\mu}(G)$. We can perform a Jordan decomposition $\lambda(g)=A_{g}^{+}-A_{g}^{-}+i\left(B_{g}^{+}-B_{g}^{-}\right)$, where $A_{g}^{+}$and $B_{g}^{+}$are positive elements. By removing a $G$ invariant conull subset from $\Lambda^{\prime}$, we can assume that $\left(\left(A_{g}^{+}\right)^{\alpha}\right)^{1 / 2} \xi_{x}^{\alpha},\left(\left(B_{g}^{+}\right)^{\alpha}\right)^{1 / 2} \xi_{x}^{\alpha},\left(A_{g}^{+}\right)^{\alpha} \xi_{x}^{\alpha},\left(B_{g}^{+}\right)^{\alpha} \xi_{x}^{\alpha}$ are contained in $D\left(\left(T^{\alpha}\right)^{1 / 2}\right)$ for every $g$ in $G, \dot{x}$ in $G / N$. We again call this $G$ invariant conull subset $\Lambda$.

For explicit calculation, square root elements are not convenient. The following proposition is useful.

Proposition 7. Suppose that $\varphi_{T}(1)<\infty$. Let $\left(A^{\alpha}\right)$ in $\operatorname{End}_{\mu}(G)^{+}$be some element appearing in the Jordan decomposition of $\lambda(g)$ for some $g$ in $G$. Then

$$
\int_{\Lambda} \omega_{T^{\alpha}}\left(A^{\alpha}\right)^{1 / 2} p_{\dot{e}}\left(\left(A^{\alpha}\right)^{1 / 2}\right) d \alpha=\int_{\Lambda}\left\langle\left(T^{\alpha}\right)^{1 / 2}\left(A^{\alpha}\right) \xi_{e}^{\alpha},\left(T^{\alpha}\right)^{1 / 2} \xi_{e}^{\alpha}\right\rangle d \alpha .
$$

Proof. First we show that it is enough to prove the result for $T^{\alpha}$ bounded. The left-hand side of this equality is rewritten as $\int_{\Lambda}\left\|\left(T^{\alpha}\right)^{1 / 2}\left(A^{\alpha}\right)^{1 / 2} \xi_{\boldsymbol{e}}^{\alpha}\right\|^{2} d \alpha$. If $\varepsilon>0$ and $\xi \in D\left(\left(T^{\alpha}\right)^{1 / 2}\right)$, then

$$
\left\|\left(\left(T^{\alpha}\right)^{1 / 2}\right)_{\varepsilon} \xi\right\| \leq\left\|\left(T^{\alpha}\right)^{1 / 2} \xi\right\|
$$

and

$$
\lim _{\varepsilon \downarrow 0}\left(\left(T^{\alpha}\right)^{1 / 2}\right)_{\varepsilon} \xi=\left(T^{\alpha}\right)^{1 / 2} \xi
$$

in norm. By Lebesgue's dominant convergent theorem, the equality is valid if we can show it with $T^{\alpha}$ replaced by $\left(T^{\alpha}\right)_{\varepsilon}$ for all positive $\varepsilon$. Moreover, $\left\{T_{\varepsilon}^{\alpha}\right\}_{\alpha \in \Lambda}$ is also a positive random operator for every positive $\varepsilon$.

We assume that every $T^{\alpha}$ is bounded. We can rewrite the equality as follows:

$$
\int_{\Lambda} \operatorname{Tr}\left(T^{\alpha}\left(A^{\alpha}\right)^{1 / 2} p_{\dot{e}}\left(A^{\alpha}\right)^{1 / 2}\right) d \alpha=\int_{\Lambda} \operatorname{Tr}\left(p_{\dot{e}} A^{\alpha} T^{\alpha}\right) d \alpha .
$$


Put $\left(A^{\alpha}\right)^{1 / 2} T^{\alpha}=S^{\alpha},\left(A^{\alpha}\right)^{1 / 2}=B^{\alpha}$. Then $\left\{S^{\alpha}\right\}_{\alpha \in \Lambda}$ and $\left\{B^{\alpha}\right\}_{\alpha \in \Lambda}$ also satisfy the randomness condition, and

$$
\begin{aligned}
\int_{\Lambda} \operatorname{Tr} & \left.T^{\alpha}\left(A^{\alpha}\right)^{1 / 2} p_{\dot{e}}\left(A^{\alpha}\right)^{1 / 2}\right) d \alpha \\
& =\int_{\Lambda} \operatorname{Tr}\left(p_{\dot{e}} S^{\alpha} B^{\alpha} p_{\dot{e}}\right) d \alpha \\
& =\int_{\Lambda} \operatorname{Tr}\left(p_{\dot{e}} S^{\alpha}\left(\sum_{\dot{g} \in G / N} V^{\alpha}(g) p_{\dot{e}} V^{\alpha}(g)^{*}\right) B^{\alpha}\right) d \alpha \\
& =\int_{\Lambda} \sum_{\dot{g} \in G / N} \operatorname{Tr}\left(p_{\dot{e}} V(g)^{\alpha} S^{g^{-1} \alpha} p_{\dot{e}} B^{g^{-1} \alpha} V^{\alpha}(g)^{*}\right) d \alpha \\
& =\int_{\Lambda} \sum_{\dot{g} \in G / N} \operatorname{Tr}\left(V^{\alpha}(g)^{*} p_{\dot{e}} V^{\alpha}(g) S^{g^{-1} \alpha} p_{\dot{e}} B^{g^{-1} \alpha}\right) d \alpha \\
& =\int_{\Lambda} \operatorname{Tr}\left(S^{g^{-1} \alpha} p_{\dot{e}} B^{g^{-1} \alpha}\right) d \alpha \\
& =\int_{\Lambda} \operatorname{Tr}\left(p_{\dot{e}} B^{\alpha} S^{\alpha}\right) d \alpha \\
& =\int_{\Lambda} \operatorname{Tr}\left(p_{\dot{e}} A^{\alpha} T^{\alpha}\right) d \alpha .
\end{aligned}
$$

By this lemma, we have

$$
\varphi_{T}(\lambda(g))=\int_{\Lambda}\left\langle\left(T^{\alpha}\right)^{1 / 2} U^{\alpha}(g) \xi_{e},\left(T^{\alpha}\right)^{1 / 2} \xi_{e}\right\rangle>d \alpha .
$$

From this equality, we recover the matrix elements of $T^{\alpha}$. Since $U^{\alpha}(g) \xi_{e}$ is not convenient for us, we use $U^{\alpha}\left(g^{-1}\right) \xi_{e}$ instead. Clearly, we have $U^{\alpha}\left(g^{-1}\right) \xi_{e}$ $=\overline{\langle\alpha, n\rangle} \xi_{g}$ where $g=n \cdot p(n \neq N, p \in X)$. This shows that

$$
\varphi_{T}\left(\lambda(n, p)^{-1}\right)=\int_{\Lambda} \overline{\langle\alpha, n\rangle} T_{e, g}^{\alpha} d \alpha .
$$

By these arguments, we have the following theorem.

THEOREM. For a positive random operator $\left\{T^{\alpha}\right\}_{\alpha \in \Lambda}$, we can recover the matrix elements $T_{e, x}^{\alpha}$ from the corresponding positive definite function $f(g)$ by

$$
T_{e, x}^{\alpha}=\sum_{n \in N} f\left((n, x)^{-1}\right) \overline{\langle\alpha, n\rangle} .
$$

General matrix elements $T_{x, y}^{\alpha}$ are obtained from the above special form by the randomness condition. We will not write down the explicit form because these are complicated by the appearance of tedious cocycles in general. 


\section{References}

[1] A. Connes, 'Sur la theorie non commutative de l'integration' (pp. 19-143, Lecture Notes in Math., vol. 725, Springer-Verlag).

[2] A. L. T. Paterson, 'A transform for Fourier algebras of the Heisenberg group', preprint.

[3] G. K. Pedersen and M. Takesaki, 'The Radon Nikodym theorem for von Neumann algebras', Acta Math. 130 (1973), 53-88.

[4] C. E. Sutherland, 'Cartan subalgebras, transverse measures and non-type I Plancherel formulae', J. Funct. Anal. 60 (1985), 281-308.

Department of Mathematics

College of Liberal Arts and Sciences

Okayama University

Tsushima, Okayama

Japan 